\title{
Performances of Estimating Null Values using Noble Evolutionary Algorithm (NEAs) by Generating Weighted Fuzzy Rules
}

\author{
Muhammad Firoz Mridha \\ Department of Computer Science \\ Stamford University Bangladesh \\ Dhaka, Bangladesh
}

\author{
Manoj Banik \\ Department of Computer Science and Engineering \\ Ahsanullah University of Science and \\ Technology, Dhaka, Bangladesh
}

\begin{abstract}
This paper Present a noble technique to estimate null values from relational database systems. At present some methods exist to estimate null values from relational database systems. The estimated accuracy of the existing methods are not good enough. We have used an advance technique for estimating null values in relational database systems. In our paper we present the technique to generate weighted Fuzzy rules from relational database systems for estimating null values using Noble Evolutionary algorithms. The parameters (operators) of the Evolutionary algorithms are adapted via Fuzzy systems. We have fuzzified the attribute values using membership functions shape. The results of the evolutionary algorithms are the weights of the attributes. The different weights of attribute generate a set of Fuzzy rules. From this we have obtained a set of rules. Our proposed techniques have a higher average estimated accuracy rate and able to estimate the null values in relational database systems.
\end{abstract}

\section{Keywords}

Fuzzy System, Membership Functions, Noble Evolutionary algorithms, Null values, Relational Database Systems, Weighted Fuzzy Rules.

\section{INTRODUCTION}

Fuzzy systems have become popular components of consumer products because they are able to solve difficult nonlinear control problem, exhibit robust behavior and present linguistic representations. These rule-based systems are more suitable for complex system problems where it is very difficult to describe the system mathematically. One of the most important considerations in designing any fuzzy system is the generation of the fuzzy rules as well as membership functions for each fuzzy set. This paper present NEAs approach to solve problem. The solving procedure mainly based on Evolutionary algorithms. It has been observed that there are many drawbacks in the early methods in estimating null values. We have estimated null values more accurately as well as to overcome the drawbacks of the previous methods. Global optimization problems are very difficult to solve. In order to understand the difficulties it is important to note that all local optimization techniques can at most locate a local minimum.

\section{FUZZY EXPERT SYSTEM}

The fuzzy expert system works as follows [1]: 1).Determine the fuzzy membership values activated by the inputs.2). Determine which rules are fired in the rule set. 3). Combine the membership values for each activated rule using the AND operator.4). Trace rule activation membership values back through the appropriate output fuzzy membership functions.5). Utilize defuzzification to determine the value for each output variable. 6). Make decision according to the output values.

\subsection{Membership Functions}

A membership function is a curve that defines how each point in the input space is mapped to a membership value as shown in Fig. 1 and Fig 2.

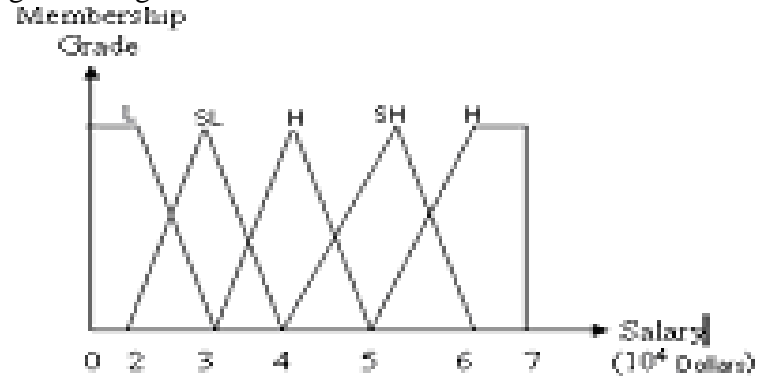

Fig 1: Membership function for salary attributes

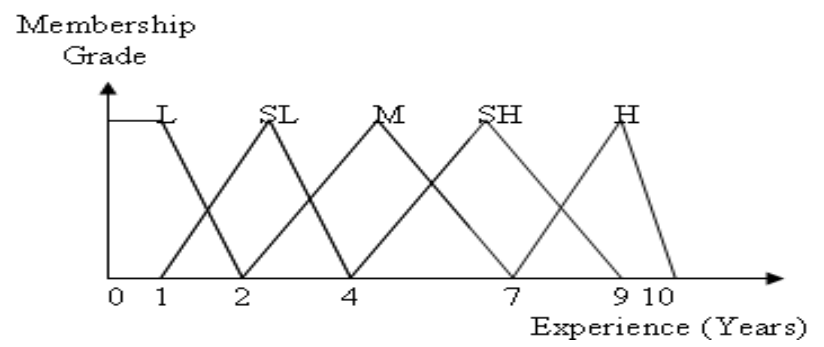

Fig.2 Membership function for Experience attributes

In this paper, the membership functions of the linguistic term, "L" ,"SL ","M","SH" ,and "H" of the attributes and "Experience" in the relational database system are adopted as shown in Fig. 2[3].

2.2 Fuzzy Rule Base

The general form of a fuzzy rule in a fuzzy system is [1]

If $\mathrm{x}_{11}$ is $\mathrm{S} 1$, and $\mathrm{x} 2$ is $\mathrm{S} 2, \ldots \ldots \ldots, \mathrm{x} \mathrm{k}$ is $\mathrm{Sk}$

Then $\mathrm{y}_{11}$ is $\mathrm{T} 1, \ldots \ldots$, and $\mathrm{y} 1$ is $\mathrm{T} 1$

\subsection{Weighted Fuzzy Rules}

Weighted fuzzy rules are a set of rules including the weights of the attributes; $w_{i j}$ denotes the weight of attributes $A_{i}$ of the $i^{\text {th }}$ rule in the rule 
RULE BASE CONTAINS WEIGHTED FUZZY RULES

Rule 1: IF $A_{1}=a_{11}\left(W=w_{11}\right)$ AND $\quad A_{2}=a_{12}\left(W=w_{12}\right) \quad$ AND .........AND $A_{n}=a_{1 n}\left(W=w_{1 n}\right)$ THEN $B=t_{1}$

Rule 2: IF $A_{1}=a_{21}\left(W=w_{21}\right)$ AND $\quad A_{2}=a_{22}\left(W=w_{22}\right) \quad$ AND .........AND $A_{n}=a_{2 n}\left(W=w_{2 n}\right)$ THEN $B=t_{2}$

Rule m: IF $A_{1}=a_{m 1}\left(W=w_{m 1}\right)$ AND $A_{2}=a_{m 2}\left(W=w_{m 2}\right)$ AND .........AND $A_{n}=a_{m n}\left(W=w_{m n}\right)$ THEN $B=t_{m}$

\subsection{BASIC CONCEPT OF FUZZY SETS:}

In a fuzzy set, each element of the set is associated with a membership value between 0 and 1 described by a membership function to indicate the grade of membership of the element in the fuzzy set. There are two types of membership to represent fuzzy sets. One is the discrete type membership function, and the other is the continuous type membership function. Let $\mathrm{U}$ be the universe of discourse, $U=\left\{u_{1}, u_{2}, \ldots, u_{n}\right\}$. A fuzzy set universe of discourse $\mathrm{U}$ can be represented as follows:

$$
\mathrm{A}=\mu_{\mathrm{A}}\left(\mathrm{u}_{1}\right) / \mathrm{u}_{1+} \mu_{\mathrm{A}}\left(\mathrm{u}_{2}\right) / \mathrm{u}_{2+\ldots \ldots \ldots \ldots . .+} \mu_{\mathrm{A}}\left(\mathrm{u}_{\mathrm{n}}\right) / \mathrm{u}_{\mathrm{n}}
$$

Where $\mu_{\mathrm{A}}$ is the membership function of the fuzzy subset A, $\mu_{A}: U->[0,1]$, and $\mu_{A}\left(u_{i}\right)$ indicate the grade of membership of $u_{i}$ in fuzzy subset $A$. If the universe of discourse $U$ is a continuous set then the fuzzy subset $A$ can be represented as follows:

$$
\mathrm{A}=/{ }_{\mathrm{u}} \mu_{\mathrm{A}}(\mathrm{u}) / \mathrm{u}, \quad \mathrm{u} \in \mathrm{U}
$$

A linguistic term can be represented by a fuzzy set represented by a membership function.

In our paper, the membership functions of the linguistic term, "L" ,"SL ","M" ,"SH" , and "H" of the attributes "Salary" and "Experience" in the relational database system are adopted as shown in Fig.1 and Fig. 2 respectively, where "L" denotes "Low", "SL" denotes "Somewhat Low", "M" denotes "Medium", "SH" denotes "Somewhat High" and "H" denotes "High".

\section{NULL VALUES}

NULL is special value for representing data we don't have. But null value is not a constant. In relational database system the attribute that has no values is defined as null values.

\section{NONLE EVOLUTIONARY ALGORITHM}

Evolutionary algorithms are specified for parameter optimization problems [5]. We used Evolutionary algorithms to generate weighted fuzzy rules.

\subsection{CROSSOVER}

In the crossover the SBMAC is used to produce the offspring population. For each subpopulation, $\tilde{\mu}$ 1offspring are generated.

\subsection{MUTATION}

In the mutation phase, the TVM operator is used to mutate all variables of an offspring [5].

\subsection{Pseudo-code structure of NOBLE EVOLUTIONARY Algorithm}

The general pseudo-code type structure of the noble evolutionary algorithm is shown below[5].

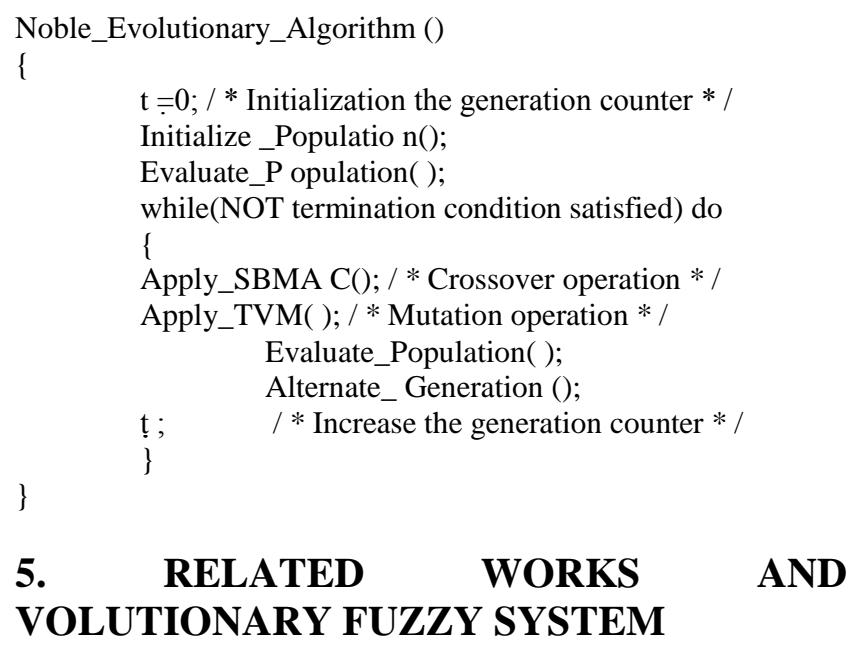

\section{RELATED WORKS AND} VOLUTIONARY FUZZY SYSTEM

In this section, we review the Chen-and-Chen's and Chen-andyeh's method for estimating null values in relational database systems [3]. First, we can use fuzzy similarity matrices to represent relations and null value can be estimated by the closeness degree of the tuple with respect to the closest rule and use expert knowledge base [3]. Fuzzy systems generally use expert knowledge base. But in complex or simple environment fuzzy systems are not designed efficiently by using expert knowledge. Moreover, for better system performance, it is more difficult and time consuming for experts to define a complete rule sets for complex system problems, which use large number of parameters. In this paper, in order to overcome the drawback of [3], we improve the methods presented in [3] and proposed a new method to estimate null values in relational database systems using Noble Evolutionary algorithm (NEAs).

\section{METHODOLOGY}

The implementation of the weighted fuzzy system is written in $\mathrm{c}++$ and complied using the Borland c++ 4.5 compilers.

\subsection{Attributes Weight Calculation}

We represent a method to calculate the weight of attributes using NEAs for estimating null values in relational database systems.

\section{FORMAT OF A CHROMOSOME}

Let us consider a relation of a relational database shown in Table 2 Based on Fig 1 and Fig 2, the values of attributes "Degree" and "Experience" shown in Table I can be fuzzified into in Table II. First, we find the format of chromosome as shown in Fig3. where the value of each gene in chromosome as a real value between zero and one and $13^{\text {th }}$ gene labeled B-L shown in Fig. 4 denotes the fuzzified values of the attributes "Degree" and "Experience" are "Bachelor" (B) and "Low" (L) respectively (e.g.. the tuples whose EMP-ID are S6 and S17 as shown in Table3. 
Generation Verses Real Value

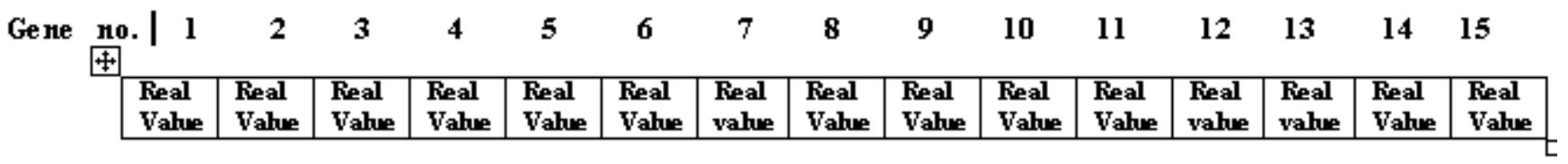

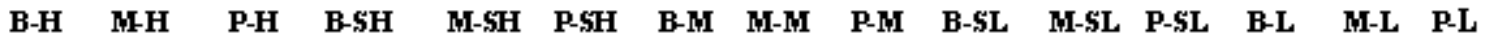
Fig: 3: Format of a chromosome

\begin{tabular}{|c|c|c|c|c|c|c|c|c|c|c|c|c|c|c|}
\hline Sene no. & 2 & 3 & 4 & 5 & 6 & 7 & 8 & 9 & 10 & 11 & 12 & 13 & 14 & 15 \\
\hline 0.017 & 0.069 & 0.386 & 0543 & 0.505 & 0.404 & 0.495 & 0.089 & 0.667 & 0.778 & 0.404 & 0.858 & 0.858 & 0.687 & 0.435 \\
\hline B-H & $\mathrm{MLH}$ & P-H & B-SH & M-SH & $\begin{array}{l}\text { P-SH } \\
\text { Fig4: }\end{array}$ & $\begin{array}{l}\text { B-M } \\
\text { Example }\end{array}$ & $\begin{array}{c}M M \\
\text { e of a c }\end{array}$ & $\begin{array}{l}\text { P-M } \\
\text { romo }\end{array}$ & & MLSL & P-SL & B-L & MLL & P-L \\
\hline
\end{tabular}

TABLE I: Relational database with Null vahues

\begin{tabular}{|c|c|c|c|}
\hline $\begin{array}{l}\text { EMIP- } \\
\text { ID }\end{array}$ & DEGREE & EXPERIENCE & SALARY \\
\hline E1 & Ph.D & 7.2 & 63000 \\
\hline $\mathrm{E2}$ & Master & 2.0 & 37000 \\
\hline E3 & Bachelor & 7.0 & 40000 \\
\hline $\mathrm{E} 4$ & Ph.D & 1.2 & 47000 \\
\hline E5 & Mlaster & 7.5 & 53000 \\
\hline E6 & Bachelor & 1.5 & 26000 \\
\hline E7 & Bachelor & 2.3 & 29000 \\
\hline E8 & Ph.D & 2.0 & 50000 \\
\hline E9 & Ph.D & 3.8 & 54000 \\
\hline E10 & Bachelor & 3.5 & 35000 \\
\hline E11 & Waster & 3.5 & 40000 \\
\hline E12 & Wlaster & 3.5 & 41000 \\
\hline E13 & Wlaster & 10.0 & 68000 \\
\hline E14 & Ph.D & 5.0 & 57000 \\
\hline E15 & Bachelor & 5.0 & 36000 \\
\hline E16 & Waster & 6.2 & 50000 \\
\hline E17 & Bachelor & 0.5 & 23000 \\
\hline E18 & Master & 7.2 & 55000 \\
\hline E19 & Wlaster & 6.5 & 51000 \\
\hline E20 & Ph.D & 7.8 & 65000 \\
\hline E21 & Master & 8.1 & 64000 \\
\hline E22 & Ph.D & 8.5 & NULL \\
\hline
\end{tabular}

From Fig.3 we can see that each chromosome represents a combination of the weights of attributes, and it is a string of the attributes which will be used to estimate null values in relational database systems. A population contains a set of chromosomes, and we can arbitrary set the number of chromosomes in a population. In this chapter, we let a chromosome consist of 15 genes. Because the total weights of the attributes must be equal to one, the weight of attribute "Experience" must equal to one minus the weight of attribute "Degree". For example, we assume that there is a chromosome as shown in Fig4. Assume that we want to estimate the null value of the attribute "Salary" of a tuple whose fuzzified value of the attributes are "Degree" and "Experience" are "Ph.D." (P) And "Some what low" (SL) then from Fig.4 we can see that the values of the gene labeled

(P-SL) is 0.858 and 0.131 (i.e., $1-0.858=0.142$ ), respectively, to calculate the degrees of closeness between the tuple which contains the null value and other tuples in the database, respectively. Therefore, the contents of the chromosome shown in Fig. 5 can be translated $n$ the following 15 rules.
TABLE II: Fuxwified vabue of Degree and Experienoe

\begin{tabular}{|c|c|c|c|}
\hline $\begin{array}{l}\text { EMP- } \\
\text { ID }\end{array}$ & DEGREE & EXPEFIEHCE & SALART \\
\hline E1 & Ph.Dn 0 & 09 & 63000 \\
\hline $\mathrm{E} 2$ & Mastern 0 & 05 & 37000 \\
\hline E3 & Bachebr/10 & 10 & 40000 \\
\hline E4 & Ph.Dn 0 & 09 & 47000 \\
\hline ES & Mastern 0 & 0.75 & 53000 \\
\hline E6 & Bachebrn 0 & 0.75 & 26000 \\
\hline E7 & Bachebr/10 & 0.65 & 29000 \\
\hline $\mathrm{E} 8$ & Ph.DM 0 & 05 & 50000 \\
\hline $\mathrm{Eg}$ & Ph.Dn 0 & 06 & 54000 \\
\hline E10 & Bachebrin 0 & 0.75 & 35000 \\
\hline E11 & Mastern 0 & 0.75 & 40000 \\
\hline $\mathrm{E} 12$ & Mastern 0 & 0.7 & 41000 \\
\hline E13 & Mastem 0 & 10 & 68000 \\
\hline E14 & Ph.DA 0 & 10 & $5 \pi 000$ \\
\hline E15 & Bachebrino & 10 & 36000 \\
\hline E16 & Master/10 & 06 & 50000 \\
\hline E17 & Bachebr/10 & 10 & 23000 \\
\hline E18 & Mastern 0 & 09 & 55000 \\
\hline E19 & Mastem 0 & 0.75 & 51000 \\
\hline E20 & Ph.Dn 0 & 06 & 65000 \\
\hline E21 & Master/10 & 055 & 64000 \\
\hline $\mathrm{E} 22$ & Ph.Dn 0 & 0.75 & 70000 \\
\hline
\end{tabular}

Rule 1: If Degree $=$ Bachelor AND Experience $=$ High, THEN the weight of degree $=0.017$ AND the weight of Experience $=0.983$.

Rule 2: If Degree=Master AND Experience=High, THEN the weight of degree $=0.069$ AND the weight of Experience $=0.931$.

Rule 3: If Degree=Ph.D. AND Experience=High, THEN the weight of degree $=0.386$ AND the weight of Experience $=0.614$.

Rule 4: If Degree=Bachelor AND Experience=Somewhat High, THEN the weight of degree $=0.543$ AND the weight of Experience $=0.457$.

Rule 5: If Degree=Master AND Experience=Somewhat High, THEN the weight of degree $=0.505$ AND the weight of Experience $=0.495$.

Rule 6: If Degree=Ph.D. AND Experience=Somewhat High, THEN the weight of degree $=0.404$ AND the weight of Experience $=0.596$ 
Rule 7: If Degree=Bachelor AND Experience=Medium, THEN the weight of degree $=0.495$ AND the weight of Experience $=0.505$.

Rule 8: If Degree=Master AND Experience=Medium, THEN the weight of degree $=0.089$ AND the weight of Experience $=0.911$.

Rule 9: If Degree=Ph.D. AND Experience=Medium, THEN the weight of degree $=0.667$ AND the weight of Experience $=0.333$.

Rule 10: If Degree $=$ Bachelor AND Experience $=$ Somewhat Low, THEN the weight of degree $=0.778$ AND the weight of Experience $=0.222$.

Rule 11: If Degree=Master AND Experience=Somewhat Low, THEN the weight of degree $=0.404$ AND the weight of Experience $=0.596$.

Rule 12: If Degree=Ph.D. AND Experience=Somewhat Low, THEN the weight of degree $=0.858$ AND the weight of Experience $=0.142$.

Rule 13: If Degree $=$ Bachelor AND Experience=Low, THEN the weight of degree $=0.858$ AND the weight of Experience $=0.142$.

Rule 14: If Degree=Master AND Experience=Low, THEN the weight of degree $=0.687$ AND the weight of Experience $=0.313$.

Rule 15: If Degree=Ph.D. AND Experience=Low, THEN the weight of degree $=0.435$ AND the weight of Experience $=0.565$.

\section{SUMMARY OF OUR METHOD}

\subsection{RULE BASE:}

A rule base is used to indicate relationships in which some attributes determines other attributes. For example, Table I shows a set of rules including the weights of the attributes, where all rules in the rule base are given by experts, $\mathrm{w}_{\mathrm{ij}}$ denotes the weight of attributes $A_{j}$ of the ith rule in the rule base, $w \in[0,1], 1 \leq i \leq n$, and $1 \leq \mathrm{j} \leq \mathrm{n}$.

\subsection{ESTIMATING NULL VALUES:}

The basic idea of our method is rule base shown in the Table I is closed to the tuple having a null value. The null value can be estimated by the closeness degree of the tuples with respect to the closest rule. Assume that, there is a relation in a relational database system having attributes $A_{1}, A_{2}, \ldots \ldots \ldots, A_{n}$ and $B$, and assume that the attributes $A_{1}, A_{2}, \ldots \ldots \ldots .$. , and $A_{n}$ determine the attribute $B$. Let " $r_{j} . A_{k}$ " denote the value of attribute $A_{k}$ appearing in the antecedent portion of rule $r_{j}$ and let " $T_{i} \cdot A_{k}$ " denote the value of attribute $A_{k}$ of tuple $T_{i}$. If there is a null value in the attribute $B$, then the value can be estimated as follows.

If the attribute $\mathrm{B}$ is defined in a numerical domain, and there rule $r_{j}$, where $1 \leq i \leq m$, in the base shown as follows:

IF $\mathrm{A}_{1}=\mathrm{a}_{\mathrm{j} 1}\left(\mathrm{~W}=\mathrm{w}_{\mathrm{j} 1}\right) \quad$ AND $\quad \mathrm{A}_{2}=\mathrm{a}_{\mathrm{j} 2}\left(\mathrm{~W}=\mathrm{w}_{\mathrm{j} 2}\right) \quad$ AND AND

$\mathrm{A}_{\mathrm{n}}=\mathrm{a}_{\mathrm{jn}}\left(\mathrm{W}=\mathrm{w}_{\mathrm{jn}}\right) \quad$ THEN $\mathrm{B}=\mathrm{N}_{\mathrm{j}}$

\subsection{CALCULATION OF THE FITNESS FUNCTION}

The fitness function measures the performance of the system. In the following, we present a method to calculate the degree of closeness between two tuples. The ranks of the terms:

\author{
Rank (Bachelor) $=10$, \\ Rank (Master) $=20$, \\ Rank (Ph.D) $=30$.
}

Suppose $\mathrm{X}$ be a nonnumeric attribute. The degree of the closeness Closeness $\left(T_{i}, T_{j}\right)$ between two tuples $T_{i}$ and $T_{j}$ can be calculated by the following rules

$$
\text { If } \operatorname{Rank}\left(\mathrm{T}_{\mathrm{i}} \cdot \mathrm{X}\right)>=\operatorname{Rank}\left(\mathrm{T}_{\mathrm{j}} \cdot \mathrm{X}\right) \text { then }
$$

$$
\begin{aligned}
\operatorname{Closeness}\left(\mathrm{T}_{\mathrm{i}}, \mathrm{T}_{\mathrm{j}}\right) & =\operatorname{Similarity}\left(\mathrm{T}_{\mathrm{i}} \cdot \mathrm{X}, \mathrm{T}_{\mathrm{j}} \cdot \mathrm{X}\right) \times \operatorname{Weight}\left(\mathrm{T}_{\mathrm{j}} \text {. Degree }\right)+ \\
& \frac{\text { Ti.Experie nce }}{\text { Tj.Experie } n c} \times \operatorname{Weight}\left(\mathrm{T}_{\mathrm{j}} \text {.Experience }\right)
\end{aligned}
$$

Where Similarity $\left(\mathrm{T}_{\mathrm{i}} \cdot \mathrm{X}, \mathrm{T}_{\mathrm{j}} \mathrm{X}\right)$ denotes the degree of similarity between $\left(T_{\mathrm{i}} \cdot \mathrm{X}\right)$ and $\left(\mathrm{T}_{\mathrm{j}} \cdot \mathrm{X}\right)$, and its value is obtained from a fuzzy similarity matrix of the linguistic terms of the attribute $\mathrm{X}$ defined by a domain expert.

Suppose that $T_{i}, T_{j}, T_{k}$ be three tuples in a relational database. Assume that the degree of closeness between tuple $T_{i}$ and $T_{j}$ is denoted as Closeness $\left(T_{i}, T_{j}\right)$ and the degree of closeness between tuple $T_{i}$ and $T_{k}$ is denoted as Closeness $\left(T_{i}, T_{k}\right)$ After calculating the degree of closeness of the other tuples in the database with respect to $T_{i}$, the system will pick a tuple which is closet to tuple $\mathrm{T}_{\mathrm{i}}$, then we can calculate the estimated value "E. $\mathrm{T}_{\mathrm{i}}$. Salary " of the attribute "Salary" of the tuple $\mathrm{T}_{\mathrm{i}}$ as follows:

$$
\text { E. } T_{i} \cdot \text { Salary }=T_{i} \cdot \text { Salary } \times \text { Closeness }\left(T_{i}, T_{j}\right)
$$

Where $T_{i}$. Salary denotes the value of the attribute "Salary" of the tuple $\mathrm{T}_{\mathrm{j}}$.

We can calculate the Errors in the following way

$$
\text { Error }=\frac{\text { E.Ti } \cdot \text { Salary }-\mathrm{T} \boldsymbol{\imath} \cdot \text { Salary }}{\mathrm{T} \boldsymbol{\imath} \cdot \text { Salary } \cdot}
$$

Now average estimated Error of the tuples based on the combination of weights of the attributes derived from the chromosome, where

$$
\operatorname{Avg\_ Error}=\left(\sum_{\mathrm{i}=1}^{\mathrm{n}} \text { Error }_{\mathrm{i}}\right) / \mathrm{n} \text {. }
$$

Then we can obtain the fitness degree of this chromosome as follows:

$$
\text { Fitness Degree }=1 \text {-Avg_Error. }
$$

\subsection{Encoding Method}

Fig.5 and Fig.6 shows the NES coding in detail. Each individual consists of two parts. This involves the Gaussians of all antecedents [1]. 


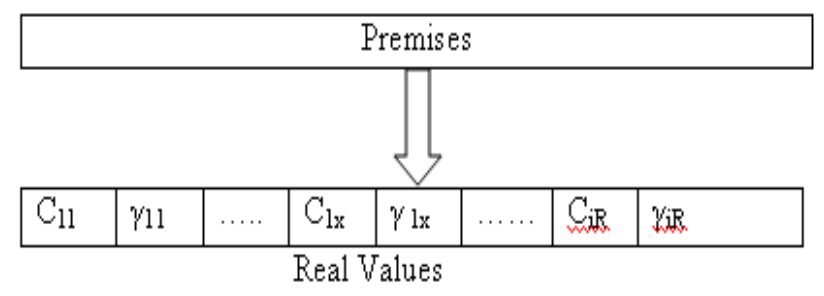

Fig 5: NES encoding

\begin{tabular}{|c|c|c|c|c|c|}
\hline \multicolumn{2}{|c|}{ Degree } & \multicolumn{2}{c|}{ Experience } & \multicolumn{2}{c|}{ Weight of Degree } \\
\hline C & W & C & W & C & W \\
\hline Rule 1 & Rule 2 & $\ldots \ldots \ldots \ldots \ldots$ & Rule n \\
\hline
\end{tabular}

Where input (Degree, Experience) and ' $C$ ' centre 'W' width

Fig 6: Fuzzy model encoding

\subsection{CROSSOVER OPERATION}

In our research we set the crossover rate $\alpha$ to 1.0. Therefore after the sections operations, the number of chromosomes in a population will continue to perform the crossover operations, where the system randomly picks up two chromosomes as the parents and randomly picks a crossover point. Then, the system performs the crossover operations on these two chromosomes at this crossover point to generate their two children.

\subsection{FUZZY RULE}

Fuzzy membership function can have different shapes. Rules obtained by simulation for Null values in relational database given Figure 7(a, b, c).

Rule 1:

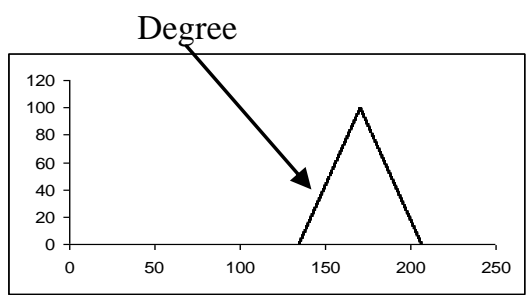

Figure 7 (a): Triangular Curve

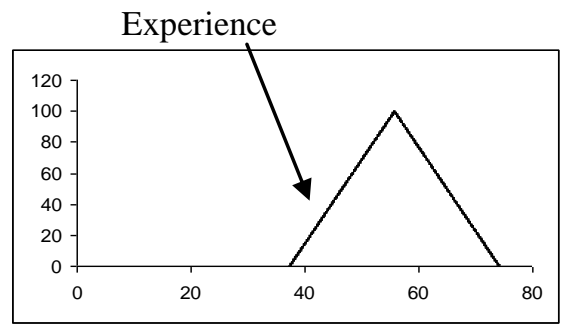

Figure 7 (b): Triangular Curve

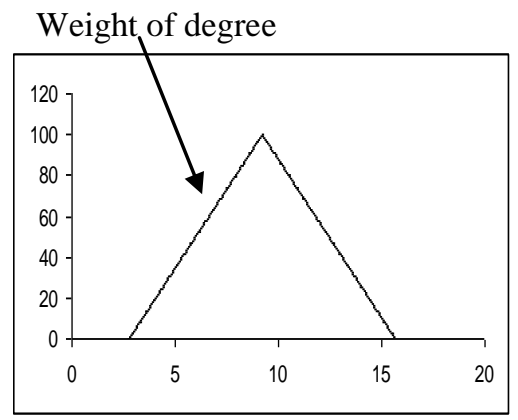

Figure 7(c): Triangular Curve

\subsection{ESTIMATED NULL VALUES IN RELATIONAL DATABASE}

In order to estimate null values in the attribute "Salary" of the tuple $T_{22}$ whose emp-id is $S_{22}$ we must find a tuple which is closest to the tuple $\mathrm{T}_{22}$. The process for computing the degree of closeness between two tuple is illustrated as follows. Let us consider the tuple $\mathrm{T}_{1}$ shown in the table whose emp id is $\mathrm{S} 1$. Both degree is Ph.D that is similar. i.e similarity $\left(\mathrm{T}_{22}\right.$. Degree, $\mathrm{T}_{1}$. Degree) $=1$. Then based on the values of the attribute "Experience" of the tuple $T_{1}$ and the tuple $T_{22}$, we can get

$$
\frac{\mathrm{T} 22 \text { Experience }}{\mathrm{T} 1 \quad \text { Experience }}=\frac{8.5}{7.2}=1.180
$$

We see that the fuzzified value of the attribute experience of the tuple T1 whose emp id is $\mathrm{S} 1$ is $\mathrm{SH}$. Therefore we pick the value of the $6^{\text {th }}$ gene label "P-SH" is 0.303 . It means that the weight of attribute Degree and Experience are 0.303 and .693 respectively. The Degree of Closeness between the tuple T22 and $\mathrm{T} 1 \mathrm{can}$ be calculated as follows

From closeness rule we get

$$
\text { Closeness } \begin{aligned}
\left(\mathrm{T}_{22}, \mathrm{~T}_{1}\right) & =1 \times 0.268+1.1180 \times(1-0.268) \\
& =0.303+0.822 \\
& =1.125
\end{aligned}
$$

After the degrees of closeness of all other tuples with respect to the tuple $T_{22}$ are calculated we can see the tuple $T_{20}$ is closest to the tuple the tuple $\mathrm{T}_{22}$, where the degree of closeness Closeness $\left(T_{22}, T_{20}\right)$ Between the tuples $T_{22}$ and $T 20$ is calculated as follows:

$$
\begin{gathered}
\text { closeness }\left(\mathrm{T}_{22}, \mathrm{~T}_{20}\right)=1 * 0.224+\frac{8.5}{7.2} *(1-0.224) \\
=0.224+1.0897 * .776 \\
=1.0698
\end{gathered}
$$

Now the salary of tuple $\mathrm{T}_{22}$ can be calculated as, follows

$$
\begin{aligned}
\mathrm{ET}_{22} & =65000 \times 1.0698 \\
& =69065.83
\end{aligned}
$$

Error of calculation

$$
\begin{aligned}
\text { Error }_{22} & =\frac{69540-70000}{70000} \\
& =-0.006
\end{aligned}
$$


TABLE III: Comparison

\begin{tabular}{|c|c|c|c|c|c|c|c|c|c|}
\hline \multicolumn{10}{|c|}{ Comparison of the estimated result of the proposed method with the existing method } \\
\hline \multirow[b]{2}{*}{ EMP_ID } & \multirow[b]{2}{*}{ Degree } & \multirow[b]{2}{*}{ Experience } & \multirow[b]{2}{*}{ Salary } & \multicolumn{2}{|c|}{$\begin{array}{l}\text { Chen-and-Chen's } \\
\text { Method[3] }\end{array}$} & \multicolumn{2}{|c|}{$\begin{array}{l}\text { Chen-and-Yeh's } \\
\text { Method[4] }\end{array}$} & \multicolumn{2}{|c|}{$\begin{array}{l}\text { Our Proposed } \\
\text { Method }\end{array}$} \\
\hline & & & & $\begin{array}{l}\text { Salary } \\
\text { Estimated }\end{array}$ & $\begin{array}{l}\text { Estimated } \\
\text { Error }\end{array}$ & $\begin{array}{l}\text { Salary } \\
\text { Estimated }\end{array}$ & $\begin{array}{l}\text { Estimated } \\
\text { Error }\end{array}$ & $\begin{array}{l}\text { Salary } \\
\text { Estimated }\end{array}$ & $\begin{array}{l}\text { Estimated } \\
\text { Error }\end{array}$ \\
\hline 1 & Ph.D. & 7.2 & 63000 & 63000 & +0.000 & 65000 & +0.032 & 62408 & -0.009 \\
\hline 2 & Master & 2 & 37000 & 33711 & -0.089 & 30704 & -0.170 & 38420 & +0.038 \\
\hline 3 & Bachelor & 7 & 40000 & 46648 & +0.166 & 35000 & -0.125 & 43200 & -0.080 \\
\hline 4 & Ph.D. & 1.2 & 47000 & 36216 & -0.229 & 46000 & -0.021 & 46029 & -0.020 \\
\hline 5 & Master & 7.5 & 53000 & 56200 & +0.060 & 54500 & +0.028 & 54125 & +0.021 \\
\hline 6 & Bachelor & 1.5 & 26000 & 27179 & +0.045 & 26346 & +0.013 & 27125 & +0.004 \\
\hline 7 & Bachelor & 2.3 & 29000 & 29195 & +0.007 & 28500 & -0.017 & 32000 & +0.103 \\
\hline 8 & Ph.D. & 2 & 50000 & 39861 & -0.203 & 50000 & +0.000 & 49845 & -0.003 \\
\hline 9 & Ph.D. & 3.8 & 54000 & 48061 & -0.10 & 55000 & +0.019 & 55042 & +0.019 \\
\hline 10 & Bachelor & 3.5 & 35000 & 32219 & -0.079 & 31538 & -0.099 & 36565 & +0.044 \\
\hline 11 & Master & 3.5 & 40000 & 40544 & +0.014 & 41590 & +0.040 & 40205 & +0.005 \\
\hline 12 & Master & 3.6 & 41000 & 41000 & +0.000 & 45159 & +0.101 & 40581 & -0.010 \\
\hline 13 & Master & 10 & 68000 & 64533 & -0.051 & 65000 & -0.044 & 67756 & -0.003 \\
\hline 14 & Ph.D. & 5 & 57000 & 55666 & -0.023 & 55000 & -0.035 & 62525 & +0.096 \\
\hline 15 & Bachelor & 5 & 36000 & 35999 & -0.000 & 35000 & -0.028 & 35241 & -0.021 \\
\hline 16 & Master & 6.2 & 50000 & 51866 & +0.037 & 48600 & -0.028 & 50457 & +0.009 \\
\hline 17 & Bachelor & 0.5 & 23000 & 24659 & +0.072 & 25000 & +0.087 & 22536 & -0.020 \\
\hline 18 & Master & 7.2 & 55000 & 55200 & +0.004 & 52400 & -0.047 & 53764 & -0.026 \\
\hline 19 & Master & 6.5 & 51000 & 52866 & +0.037 & 49500 & -0.029 & 51209 & +0.004 \\
\hline 20 & Ph.D. & 7.8 & 65000 & 65000 & +0.000 & 65000 & +0.000 & 65625 & $\begin{array}{l}+0.009 \\
\end{array}$ \\
\hline 21 & Master & 8.1 & 64000 & 58200 & -0.091 & 58700 & -0.083 & 62372 & -0.025 \\
\hline 22 & Ph.D. & 8.5 & 70000 & 67333 & -0.038 & 65000 & -0.071 & 69540 & -0.006 \\
\hline \multicolumn{3}{|c|}{ Average estimated Error } & & & +0.062 & & +0.051 & & +0.006 \\
\hline
\end{tabular}

\section{CONCLUSION}

In this paper Based on the best chromosome we have estimated the Null values of attributes "salary" where the attribute contain the different weight of degree and experience .After a predefined number of evolution of the NEA the best chromosome contain the optimal weight of the attribute and they are translated into a set of rules to be used for estimating Null values. This proposed method can get a higher average estimated accuracy rate.

\section{REFERENCES}

[1] Yuhui Shi, Russell Eberhart, Yaobin Chen, "Implementationof Evolutionary Fuzzy Systems", IEEE Transaction of Evolutionary Computation, Vol-7, No.2, April 1999.

[2] S.M.Chen and M.S.yeh."Generating fuzzy rules from relational database systems for estimating null values."Cybern.Syst.vol.28,no.8.pp.665-723,1997.

[3] S.M.chen and H.H. Chen ,"estimating Null values in the distributed relational databases environment." Cybern. Syst.,vol.31.no.8.pp,851-871,2000.

[4] H.L.Lin and S.M. Chen, “Generating weighted Fuzy rules from training data for handling fuzzy classification problems,"in
Proc.2000 Int. Computer Symp.: workshop Artificial Intelligence, Chiayi, Taiwan ,R.O.C.,2000, pp.11-18.

[5] M.M.A Hashem ,Keigo Watanabe "evolutionary Computations", Khulna University of Engineering and

Technology,Bangladesh

[6] J.C.Cubero and M.A. Vila,"A new definition of fuzzy functional dependency in fuzzy relational databases," Int.J.intel.Syst.,vol.9.no.5,pp.441-448,1994.

[7] J.grant,"Null values in a relational database," inform.Processing Lett., vol.6,no.5,pp.156-157,1977.

[8]C.M.Haung and S.M.Chen, "A new method to estimate null values in relational database systems using geneticalgorithms, "inProc. $6^{\text {th }}$ Conf.ArtificialIntelligenceAppl ications,Kaushiung,Taiwan,R.O.C.,2001,2000,pp.599-604.

[9] D.G.Burkhardt and P.P.Bonissone."Automated fuzzy knowledge base Generation and tuning." In Proc.1992 IEEE Int.Conf. Fuzzy Systems San Diego,CA.1992,pp.179-188.

[10] P.Bosc.D.Dubois,and H.Prade, "Fuzzy functional dependencies and redundancy elimination." J.Amer. Soc.inform. Sci.,vol.49.pp.217-235,1998 